\title{
Pattern Reconfigurable Antenna for VANET, Wi-Fi, and WiMAX Wireless Communication Systems
}

\author{
Rahmani Faouzi iD, ${ }^{1}$ Amar Touhami Naima, ${ }^{1}$ BelbachirKchairi Abdelmounaim ${ }^{D}{ }^{2}$ \\ Aknin Noura, ${ }^{1}$ and Taher Nihade ${ }^{3}$ \\ ${ }^{1}$ Faculty of Sciences, Abdelmalek Essaadi University, Tetouan 93030, Morocco \\ ${ }^{2}$ Faculty of Science and Technology of Mohammadia, Hassan II University of Casablanca, Casablanca 20006, Morocco \\ ${ }^{3}$ National School of Applied Sciences, Abdelmalek Essaadi University, Tetouan 93030, Morocco \\ Correspondence should be addressed to Rahmani Faouzi; rahmani.faouzi-etu@uae.ac.ma
}

Received 6 March 2021; Revised 30 September 2021; Accepted 15 October 2021; Published 31 October 2021

Academic Editor: Francesco D'Agostino

Copyright (c) 2021 Rahmani Faouzi et al. This is an open access article distributed under the Creative Commons Attribution License, which permits unrestricted use, distribution, and reproduction in any medium, provided the original work is properly cited.

\begin{abstract}
This work presents the design and analysis of a beam switching antenna for VANET, Wi-Fi, and WiMAX wireless communication systems. The proposed reconfigurable antenna is powered by a coaxial cable and consists of a circular patch, six fish-shaped radiating elements, and a circular planar ground. The antenna was constructed on a Rogers RT5880 substrate. Its dimensions are as follows: $0.81 \lambda_{0} \times 0.81 \lambda_{0} \times 0.03 \lambda_{0}$. It performs six reconfigurable operating states, at the same frequency, by controlling the activation and deactivation of six PIN diodes to change the beam's direction. A theoretical equivalent circuit model of the antenna is extracted. A progressive analysis of improving the antenna characteristic performances is provided. The bandwidth of the proposed antenna is $9.07 \%$ (measured), 9.62\% (simulated), and $9.31 \%$ (theoretical). The designed antenna has a maximum gain of $9.57 \mathrm{~dB}$ for all pattern states and a superior efficiency ratio from $85 \%$ to $95 \%$ over the operating range $(5.54 \mathrm{GHz}-6.10 \mathrm{GHz})$. The proposed reconfigurable antenna is fabricated. Measured, simulated, and theoretical results are given and show good agreement, including reflection coefficient (S11) and radiation patterns.
\end{abstract}

\section{Introduction}

In the last decade, wireless communication in different fields has been developed enormously due to the low cost and high performance of wireless devices. In the domain of antenna systems, the reconfigurable antenna design has gained a lot of attention [1-3]. Its versatility in frequency picking, polarization selectivity, and pattern reconfiguration makes it very suitable for various wireless communication systems, such as wireless WLAN systems. The reconfigurable pattern antenna can produce different radiation patterns on a physical aperture at the same operating frequency. This antenna concept can provide electrical adjustment mechanisms with multifunctional control of electromagnetic waves, such as adjusting the applied bias voltage.
Typical active elements used in reconfigurable metasurfaces $[4,5]$ include varactor diodes [6], radio-frequency micro-electromechanical systems (RF-MEMSs) [7], and PIN diodes [8], which have been used to achieve beam direction. Since they are not very costly, the PIN diodes are very effective and can easily combine with patch antennas [9-12].

There are some key approaches and strategies in the literature to explain the reconfigurability of the pattern with enhanced radiation gain and efficiency [13-16]. By monitoring the condition of two PIN diodes between the feed line and patch components, the antenna in [17] achieved two states. The radiation pattern of the antenna in [18] would be reconfigured by steering the status of two PIN diodes in two modes in the azimuth plane based on a circular monopole patch and three ship-shaped parasitic patches. The antenna 


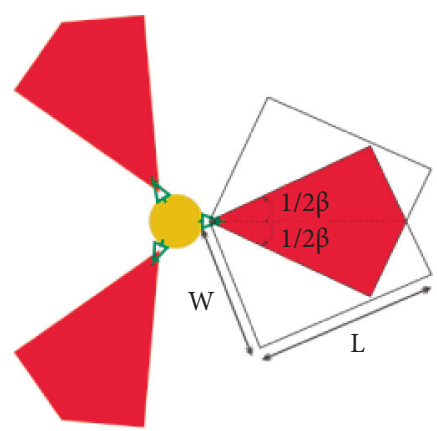

(a)

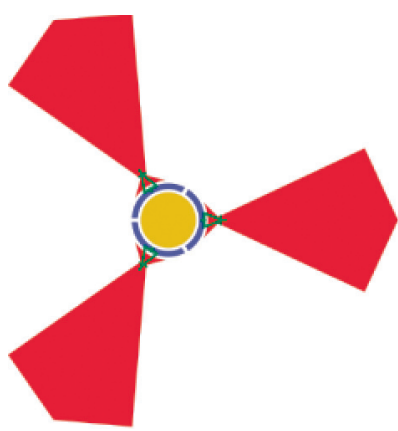

(b)

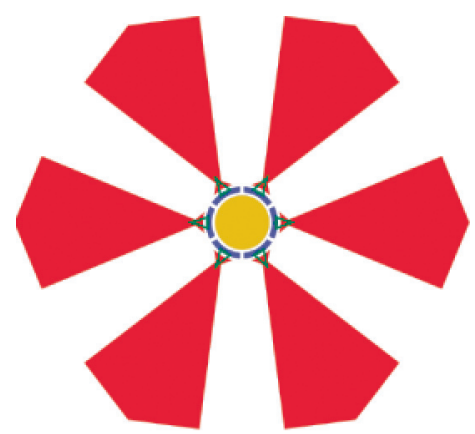

(c)

Figure 1: The antenna design evolution: (a) Ant. 1, (b) Ant. 2, and (c) Ant. 3.

in [19] monitored the status of PIN diode switches in four operating states. We propose using six radiating cells to increase the antenna performance. The goal is to increase the bandwidth and also to improve antenna gain, in addition to the pattern reconfigurability.

The novelty of the proposed design, compared to all our published works, is mainly the ability to improve the antenna performance such as gain, bandwidth, and efficiency [20-23]. A circular planar antenna with reconfigurable radiation pattern using parasitic patches was proposed in our previous study [20], but this kind of antenna was not be able to cover the whole azimuth plane. The antenna in [21] has only a bandwidth of $4.7 \%$ and a gain of $8.22 \mathrm{~dB}$ for four pattern states. In addition, a wideband reconfigurable antenna with beams switching has been proposed in [22], and it has a bandwidth of $26.75 \%$, while the gain is less than $6 \mathrm{~dB}$. A new reconfigurable antenna was proposed in [23] using eight hexagon-shaped radiation cells; the antenna has a bandwidth of $6.2 \%$ and gains of $8.46 \mathrm{~dB}$.

In this work, we propose a new antenna using six fishshaped radiating cells with reconfigurable radiation patterns that operate either as a reflector(s) or director(s) and a circular planar ground according to the switch states. Using six PIN diodes, the radiation pattern can be modified into six states. By controlling the states (ON or OFF) of six Microsemi MPP4203 PIN diodes [24], the main beam of the antenna can be redirected in six directions in half an elevation plane. The proposed antenna may also alter the direction of the main lobe in the azimuth plane at specific steering angles. The proposed design guarantees the possibility that the antenna can radiate in all directions of the $x y$-plane. The simulated realized gains for all pattern states are about $9.57 \mathrm{~dB}$. The designed reconfigurable antenna operates for VANET, Wi-Fi, and WiMAX wireless communication systems and has a simulated bandwidth of 9.62\% from $5.54 \mathrm{GHz}$ to $6.10 \mathrm{GHz}$.

The content of this work is divided into three sections. Section 1 is presented as a general introduction. In Section 2, we introduce the design principle used to realize the reconfigurability of radiation patterns, we present the parametric study of the proposed antenna that is used to obtain the optimal values and the best performance, and we discuss the obtained measurement and simulation results. Finally, the main conclusions of this study are given in Section 3.

\section{Design Principle}

Figure 1represents the proposed antenna design evolution, with corresponding results of simulated reflection coefficient (S11) as shown in Figure2(a) and simulated realized gain of each step as shown in Figure 2(b). All proposed structures are engraved on $h=1.57 \mathrm{~mm}$ thick Rogers RT5880 substrate with a relative permittivity of 2.2 and a loss tangent of 0.0009 . The antenna geometry must be chosen in such a way that the antenna can direct the main lobe in very precise directions. To cover the whole azimuth plane (xoy-plane), the feed line using coaxial cable is more adequate for the designed antenna. The proposed design is generally formed of some modified rectangular patch antennas distributed symmetrically around a coaxial feed line as shown in Figure 1(a). The antenna center frequency was designed as $5.8 \mathrm{GHz}$, and the rectangular patch physical parameters were calculated using the following equations used in the transmission line model [25].

$$
W=\frac{c}{2 f} \sqrt{\frac{2}{\varepsilon_{r}+1}},
$$

where $c$ represents the electromagnetic wave speed in free space.

$$
\begin{aligned}
L & =\frac{c}{2 f \sqrt{\varepsilon_{r e}}}-2 \Delta L, \\
\varepsilon_{r e} & =\frac{\varepsilon_{r}+1}{2}+\frac{\varepsilon_{r}-1}{2} \frac{1}{\sqrt{(1+12 h / W)}}, \\
\Delta L & =0,412 h \frac{\left(\varepsilon_{r e}+0,3\right)(W / h+0,264)}{\left(\varepsilon_{r e}-0,258\right)(W / h+0,8)} .
\end{aligned}
$$

Each radiating element is connected to the feed line via a PIN diode in the gap between them. By controlling the ON and the OFF states of the Microsemi MPP4203 PIN diodes, the distribution of the antenna surface current will be modified, so the antenna radiation direction will deviate. When a PIN diode is reverse biased, the radiating cell will act as a director, while in direct biased, the parasitic patch will act as a reflector. The initial step in the design of the proposed reconfigurable antenna is presented in Figure 1(a). As can be seen from this figure, on the top side of the substrate, 


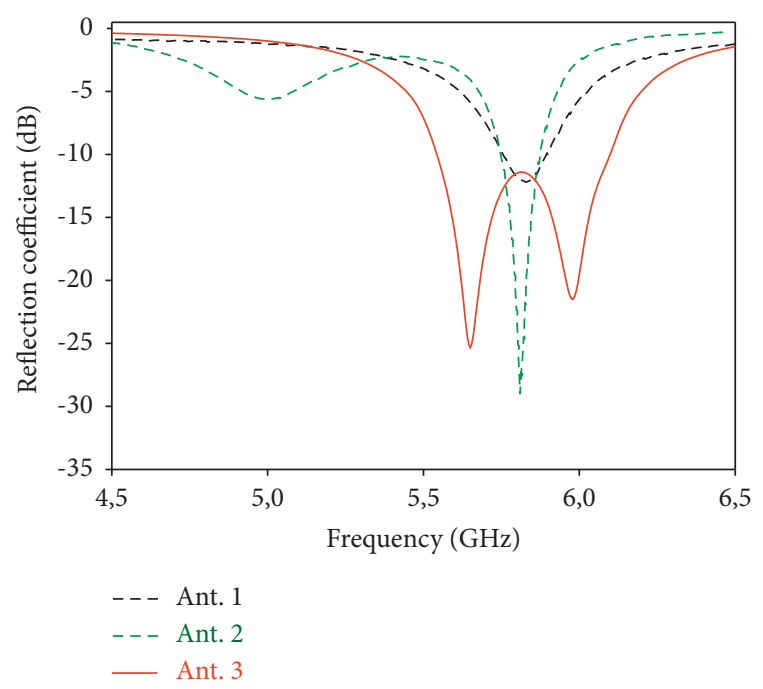

(a)

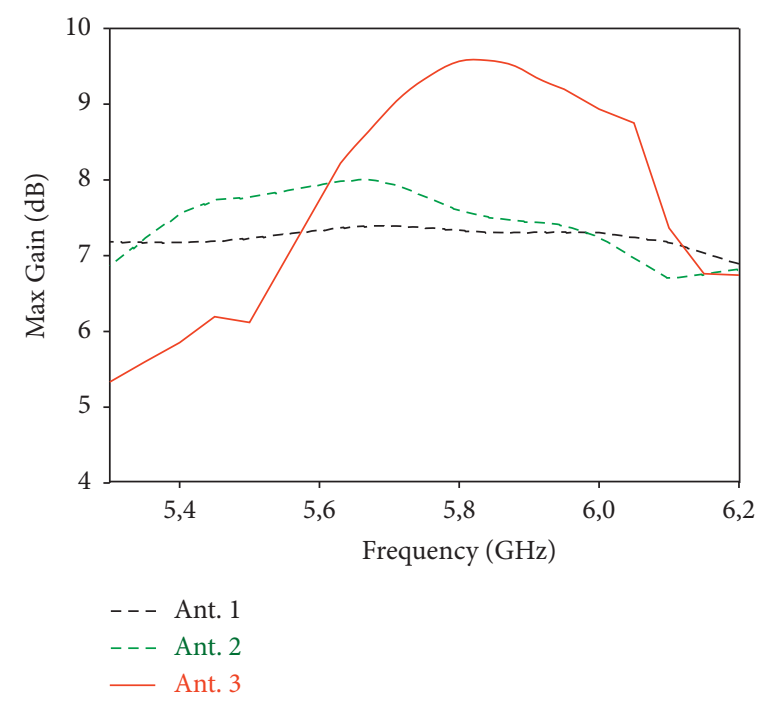

(b)

Figure 2: Reflection coefficient (a) and gain (b) for different antennas.

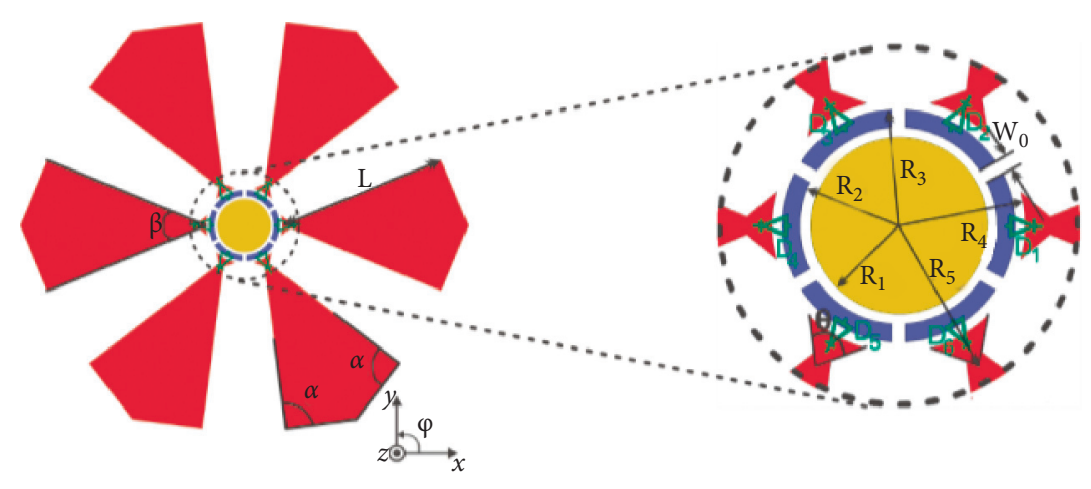

(a)

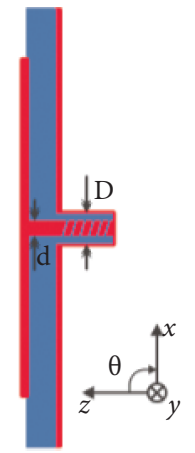

(b)

Figure 3: The designed reconfigurable antenna. (a) Top view. (b) Side view.

three symmetrical kite-shaped radiating elements are placed so that one is rotated from the other by $120^{\circ}$, and a circularshaped patch located at the center of the antenna is connected to the coaxial cable inner pin. Using three PIN diodes, the radiating elements are connected to the central circular patch, which is connected to the inner pin of the coaxial cable. As shown in Figure 2(a), the initial antenna (Ant. 1) resonates at $5.8 \mathrm{GHz}$ frequency, and the simulated $\mathrm{S} 11$ parameter value is not also below $-12 \mathrm{~dB}$. Note that the maximum simulated gain value does not exceed $7.14 \mathrm{~dB}$ as shown in Figure 2(b).

In order to make the antenna more adapted for $5.8 \mathrm{GHz}$ and to increase the antenna gain, a change in the shape of the radiating elements was implemented as shown in Figure 1(b). In the second step geometry (Ant. 2), three fanshaped coupling elements distributed symmetrically around the antenna center are added. As presented in Figure 2(a), a resonant frequency at $5.8 \mathrm{GHz}$ is created with $-10 \mathrm{~dB}$ impedance bandwidth that ranges from 5.74 to $5.86 \mathrm{GHz}$. Also, it can be seen that the maximum simulated gain value is $7.62 \mathrm{~dB}$ for Ant. 2, as presented in Figure 2(b).

In order to improve the bandwidth and also to enhance the antenna gain, in addition to the pattern reconfigurability, a change in the number of the radiating cells and coupling elements was implemented as shown in Figure 1(c). The final proposed antenna geometry is formed of three parts, as shown in Figure 3. The first part (yellow area) is a circularshaped patch located at the center which is connected to the inner pin of the coaxial cable. The second part (blue area) comprises six fan-shaped coupling elements, which are connected by six PIN diodes with six fish-shaped radiating cells (red area) distributed around the antenna center symmetrically. The substrate size which has the circularshaped is $(\varnothing \times H)=(80 \mathrm{~mm} \times 1.57 \mathrm{~mm})$.

In order to design and to simulate the proposed antenna, Computer Simulation Technology Microwave Studio (CST MWS) software was used. Table 1 represents the final optimized parameters values of the proposed antenna design. As 
TABle 1: Dimensions of antenna in Figure 3.

\begin{tabular}{lcccccc}
\hline Parameter & $L$ & $R 1$ & $R 2$ & $R 3$ & $R 4$ & $R 5$ \\
\hline Dimension & $16 \mathrm{~mm}$ & $2.5 \mathrm{~mm}$ & $2.8 \mathrm{~mm}$ & $3.3 \mathrm{~mm}$ & $3.6 \mathrm{~mm}$ & $4.6 \mathrm{~mm}$ \\
Parameter & $W 0$ & $D$ & $d$ & $\theta$ & $\alpha$ & $4^{\circ}$ \\
Dimension & $0.4 \mathrm{~mm}$ & $4.1 \mathrm{~mm}$ & $1.2 \mathrm{~mm}$ & $80^{\circ}$ & $95^{\circ}$ \\
\hline
\end{tabular}

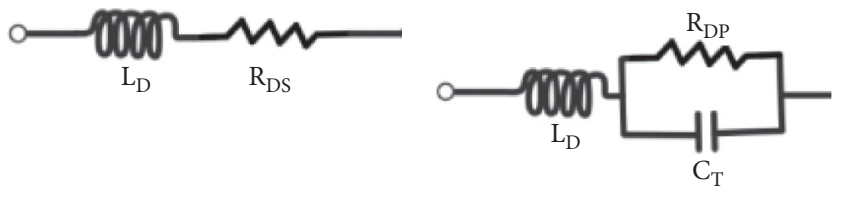

(a) (b)

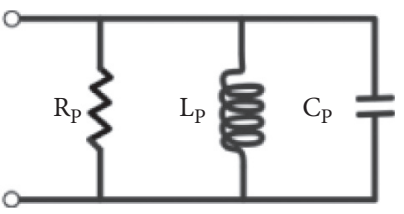

(c)

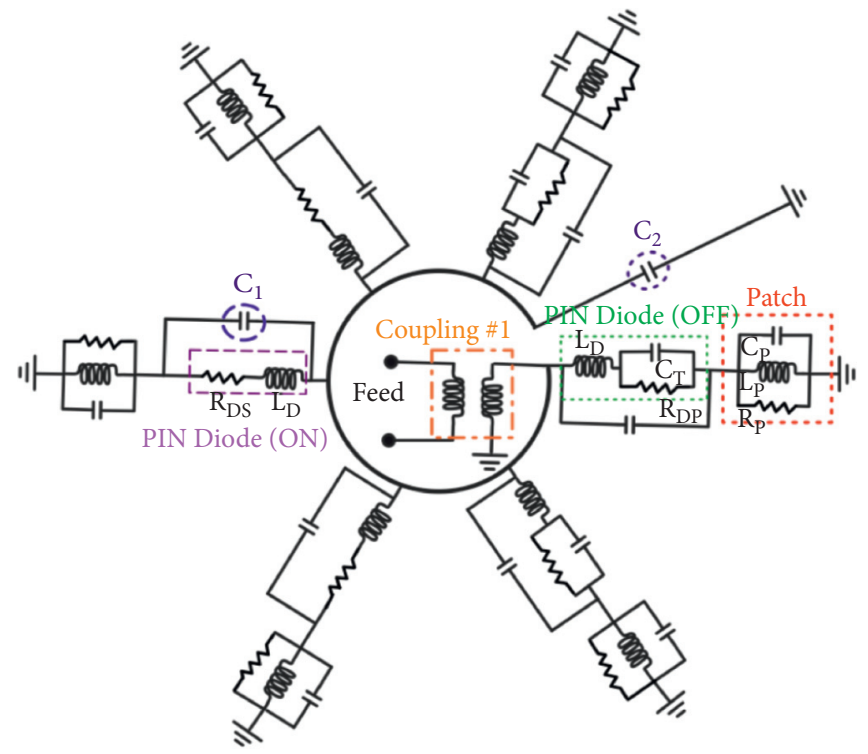

(d)

Figure 4: Equivalent circuit model. (a) Forward bias of PIN diode (ON). (b) Reverse bias of PIN diode (OFF). (c) Rectangular patch antenna. (d) Proposed antenna.

given in Figure 2(a), the final proposed reconfigurable antenna operates in the band ranging from $5.54 \mathrm{GHz}$ to $6.10 \mathrm{GHz}$ with the center frequency at $5.82 \mathrm{GHz}$. As given in Figure 2(b), the final antenna has a maximum gain of $9.57 \mathrm{~dB}$ at $5.82 \mathrm{GHz}$, greater than that of the two previous antennas. The main reason for getting suddenly high gain of a proposed antenna (Ant. 3) compared with Ant.1 and Ant. 2 is the use, for each state, of three radiating cells as directors and three other radiating cells as reflectors.

The fish-shaped radiating elements are selectively powered by the circular patch through PIN diode biasing. According to the datasheet of the Microsemi MPP4203, the diode parameters are $L_{\mathrm{D}}=0.02 \mathrm{nH}, R_{\mathrm{DS}}=3 \Omega, C_{\mathrm{T}}=0.08 \mathrm{pF}$, and $R_{\mathrm{DP}}=25 \mathrm{~K} \Omega$. The RF equivalent circuit of the diode is shown in Figures 4(a) and 4(b) for both the ON and the OFF states, respectively $[19,21,24]$. A rectangular microstrip patch is analyzed and considered as a parallel combination of resistance $\left(R_{\mathrm{P}}\right)$, inductance $\left(L_{\mathrm{P}}\right)$, and capacitance $\left(C_{\mathrm{P}}\right)$ as shown in Figure 4(c). Expressions for the lumped elements of $R_{\mathrm{P}}, L_{\mathrm{P}}$, and $C_{\mathrm{P}}[26,27]$ are given as

$$
\begin{gathered}
C_{P}=\frac{\varepsilon_{0} \varepsilon_{r e} L W}{2 h} \cos ^{-2}\left(\frac{\pi Y_{f}}{L}\right), \\
L_{P}=\frac{1}{\omega_{P}^{2} C_{P}}, \\
R_{P}=\frac{Q_{r}}{\omega C_{P}},
\end{gathered}
$$

where $L=$ length of the patch, $W=$ width of the patch, $Y_{\mathrm{f}}=y$ coordinates of feed point, and $h=$ thickness of the substrate material.

$$
Q_{r}=\frac{c \sqrt{\varepsilon_{r e}}}{4 f h} .
$$

Figured 4(d) represents the equivalent circuit of the proposed antenna. The circular gap around the central circular patch, as represented in Figure 2(a), can be 
TABLE 2: Optimized lumped parameter values.

\begin{tabular}{lcccccc}
\hline Parameter & $R_{\mathrm{P}}(\Omega)$ & $L_{\mathrm{P}}(\mathrm{nH})$ & $C_{\mathrm{P}}(\mathrm{pF})$ & $\mathrm{C}_{1}(\mathrm{pF})$ & $\mathrm{C}_{2}(\mathrm{pF})$ & $k$ \\
\hline Value & 245 & 0.41 & 1.71 & 0.04 & 0.043 & 1 \\
\hline
\end{tabular}

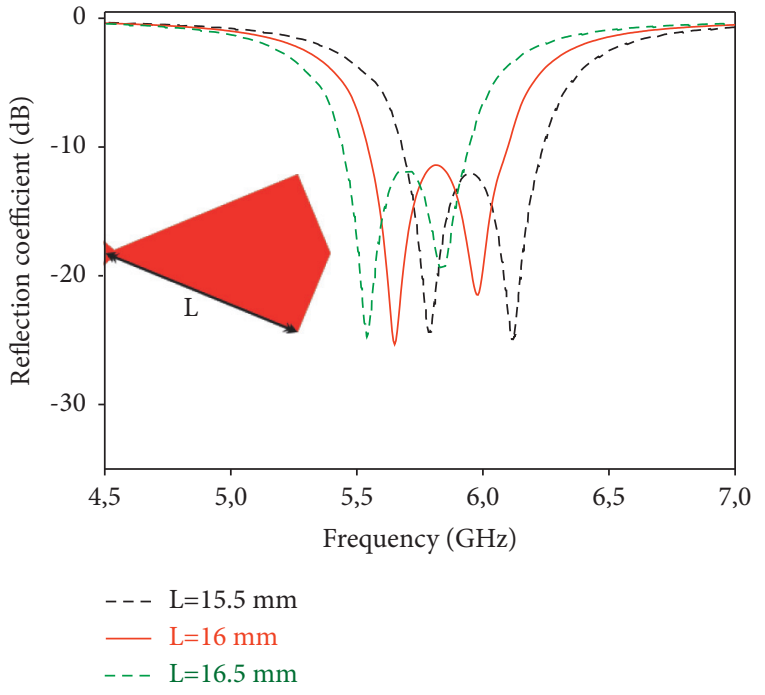

(a)

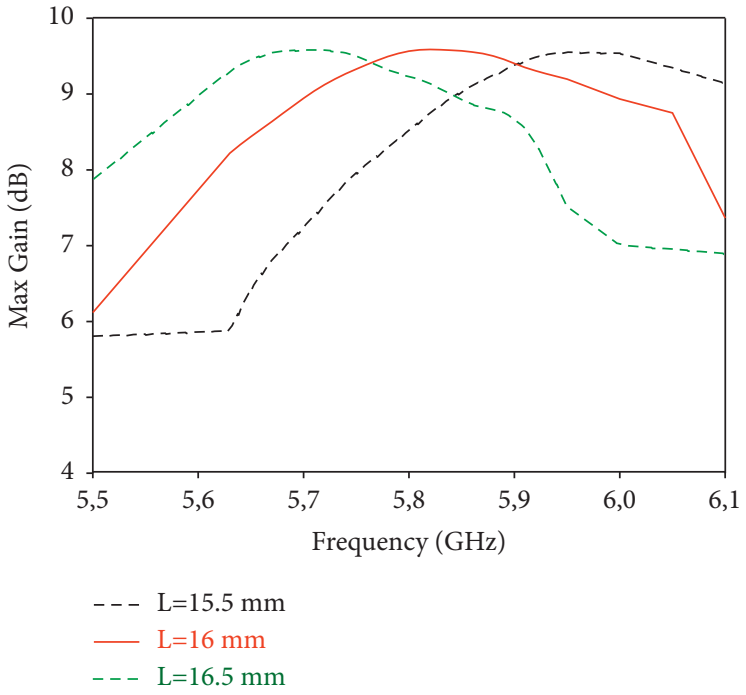

(b)

FIGURE 5: Reflection coefficient (a) and gain (b) for different length (L) values.

considered as a coupling by mutual inductance (coupling \#1) characterized by the coupling coefficient $k$. Taking into account the inter-element couplings, the gap between each coupling element (blue area) and the neighboring fishshaped radiating cell can be analyzed as a parasitic capacitance $\left(C_{1}\right)$ in parallel with the equivalent circuit of the PIN diode connected in this gap. The cumulative charges between the coupling elements and the ground can be considered as a parasitic capacitance $\left(\mathrm{C}_{2}\right)$. In order to simulate the equivalent circuit of the proposed antenna, Advanced Design System (ADS) was used. The final optimized lumped parameters values are presented in Table 2.

2.1. Parametric Study. A parametric study was conducted to comprehend the effect on the proposed antenna performance. In this section, we study the effects of the main geometric parameters on reflection coefficient (S11) and antenna gain. We present a parametric study of the proposed antenna that is used to obtain the optimal values and the best performance by tuning one parameter at a time while other parameters are left invariable. Concerning the parameters $\left(R_{2}, R_{3}, R_{4}\right.$, and $\left.\mathrm{W}_{0}\right)$, we took the optimal minimum values to fabricate the proposed antenna.

2.2. Effect of Length L. The reflection coefficient (S11) and antenna gain for different values of length $(L)$ of radiating patches are simulated and plotted in Figure 5. It can be seen that the impedance bandwidth of the proposed antenna remains stable and that the center frequency is shifted. As shown in Figure 5(a), when $L=16 \mathrm{~mm}$, the band frequency is shifted to the desired center frequency value with better adaptation, while the antenna gain becomes maximum at $5.82 \mathrm{GHz}$ when the length is $L=16 \mathrm{~mm}$, as shown in Figure 5(b). So, the optimal value of length is $L=16 \mathrm{~mm}$.

2.3. Effect of Angle $\beta$. The antenna gain and the reflection coefficient (S11) are also simulated as a function of the different angle $(\beta)$ values. As shown in Figure 6(a), the center frequency remains stable. As $\beta$ increases, the frequency band reduces. In parallel, the antenna gain becomes maximum $(9.57 \mathrm{~dB})$ at a frequency of $5.82 \mathrm{GHz}$ when the angle is $\beta=45^{\circ}$, as shown in Figure 6(b). We can see that the best result is obtained for an angle $(\beta)$ equal to $45^{\circ}$.

2.4. Effect of Angle $\theta$. The antenna gain and the S11 parameter are also simulated versus the different angle $(\theta)$ values. As shown in Figure 7(a), the center frequency remains stable. The desired frequency band is obtained for the angle $\theta=80^{\circ}$. As illustrated in Figure 7(b), the antenna gain becomes maximum at the desired frequency when the angle is $\theta=80^{\circ}$. We notice thereby that the best result is obtained for an angle $(\theta)$ equal to $80^{\circ}$.

2.5. Effect of Radius R1. In this section, we display the effect of the radius $\left(R_{1}\right)$ of the central patch on the S11 parameter and the antenna gain, while keeping the other dimensions invariable. From Figure 8(a), we show the curves of S11 parameter with different values of $R_{1}$. As the radius $R_{1}$ increases, the center frequency changes, and the frequency band moves 


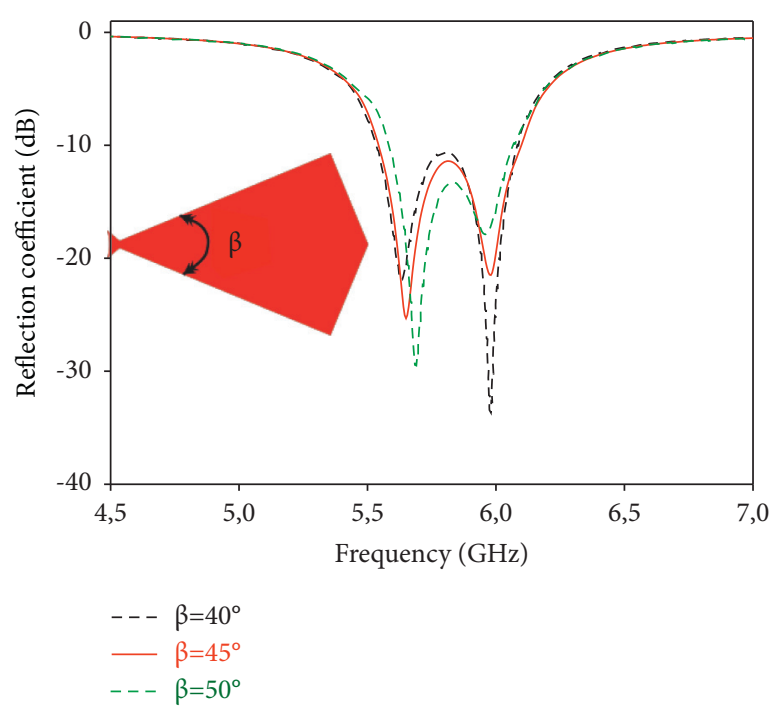

(a)

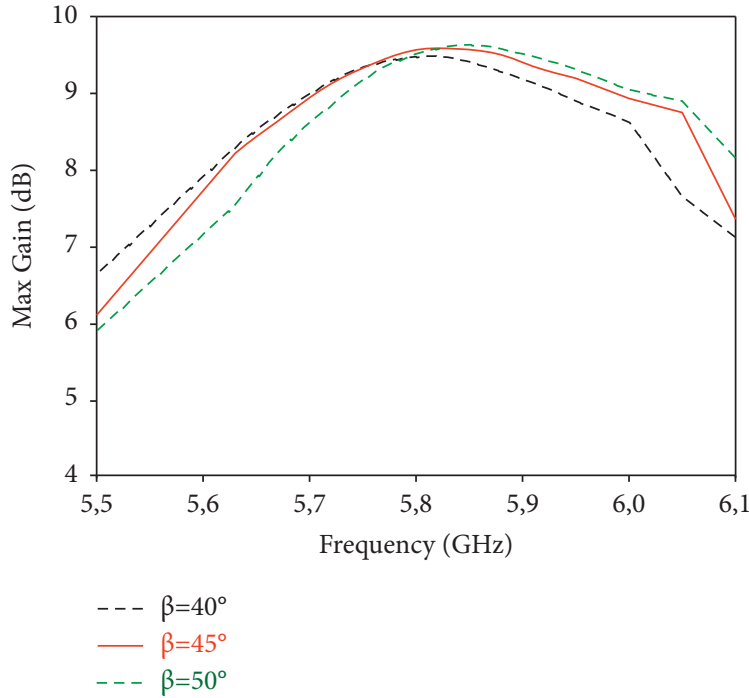

(b)

Figure 6: Reflection coefficient (a) and gain (b) for different angles $(\beta)$.

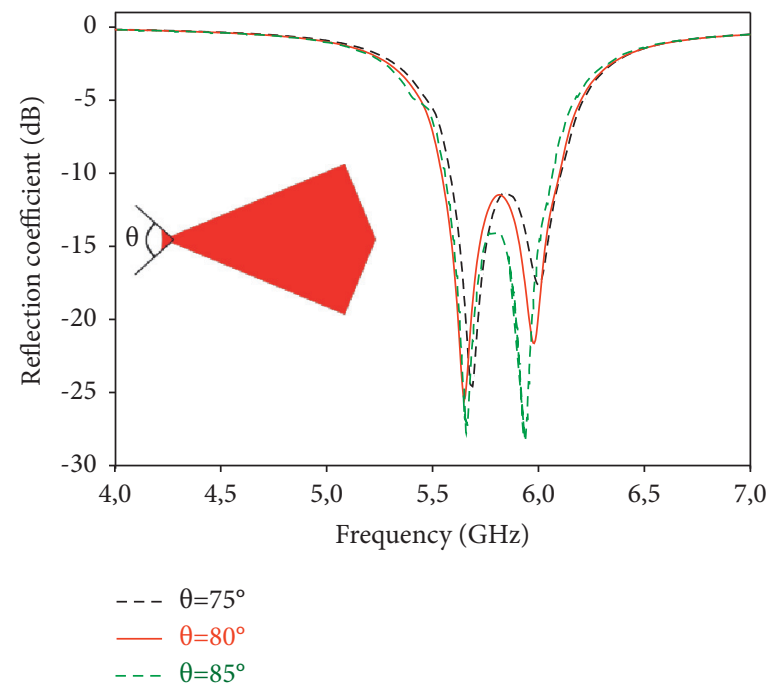

(a)

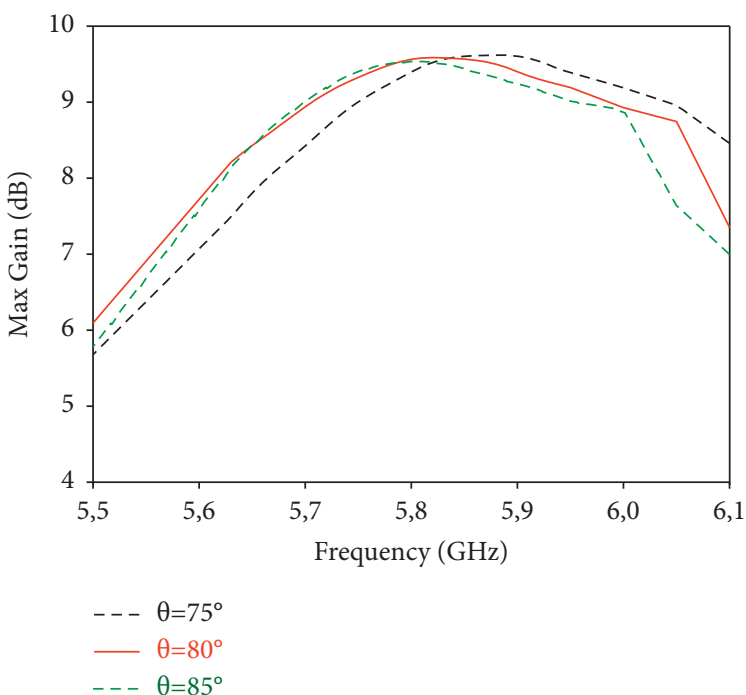

(b)

FiguRE 7: Reflection coefficient (a) and gain (b) for different angle values $(\theta)$.

to the desired value $(5.54-6.10 \mathrm{GHz})$ for $2.5 \mathrm{~mm}$ of $R_{1}$. The impedance bandwidths are about $560 \mathrm{MHz}$. On the other hand, Figure $8(\mathrm{~b})$ illustrates the simulated gain versus frequency with different values of $R_{1}$. We can see from this figure that the maximum gain value at $5.82 \mathrm{GHz}$ is obtained for $2.5 \mathrm{~mm}$ of $R_{1}$. So, the optimal value of radius $R_{1}$ is $2.5 \mathrm{~mm}$.

2.6. Experimental Results and Discussion. The antenna, which is shown in Figure 3, is fabricated using a printed circuit board (PCB) milling machine (LPKF Protomat E33), as presented in Figure 9. The control of each PIN diode is done by two biasing lines: the first one is served as a direct current (DC) supply and the other is served as a ground. In each operating mode, three PIN diodes are forward biased while the other three PIN diodes are reverse biased. By changing the distribution of the antenna surface current, this will deflect the direction of the antenna radiation. Thus, simulation series were performed as given in Table 3 . The fish-shaped radiating cell acts as a director when the PIN diode is reverse biased. Otherwise, it acts as a reflector. It shows that the proposed antenna has six operating states named as S1, S2, S3, S4, S5, and S6. Table 3 presents specific biasing requirement for these six operating states.

The S11 parameter of the proposed antenna is measured using "Rohde and Schwarz ZVB20 vector network analyzer" to verify obtained simulation results. Figure 10(a) shows the comparison of S11 plot of measured, simulated, and theoretical results of the proposed 


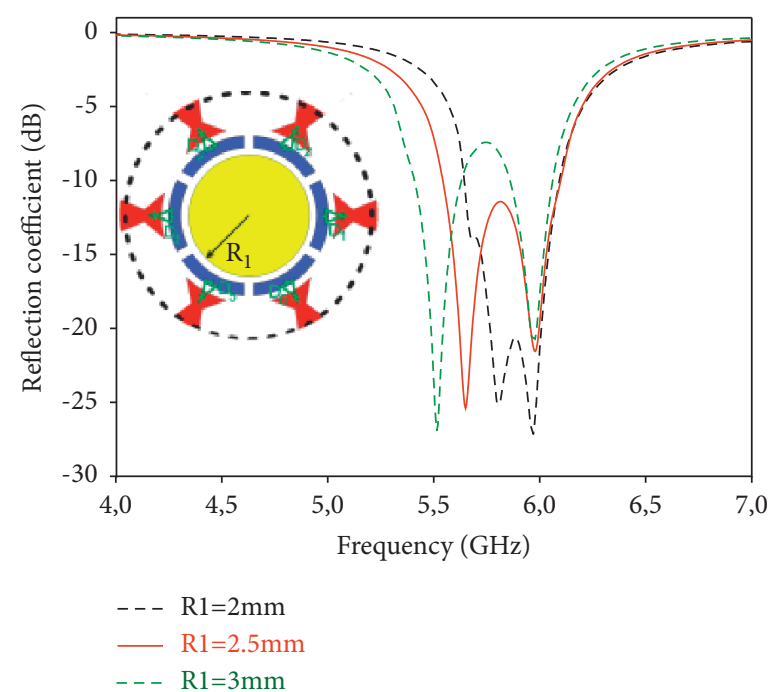

(a)

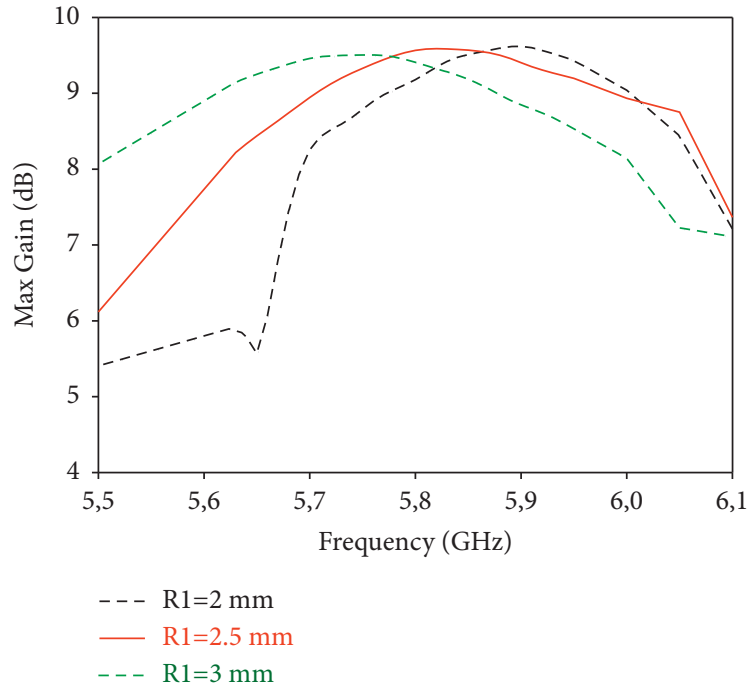

(b)

FIGURE 8: Reflection coefficient (a) and gain (b) for different radius (R1) values.

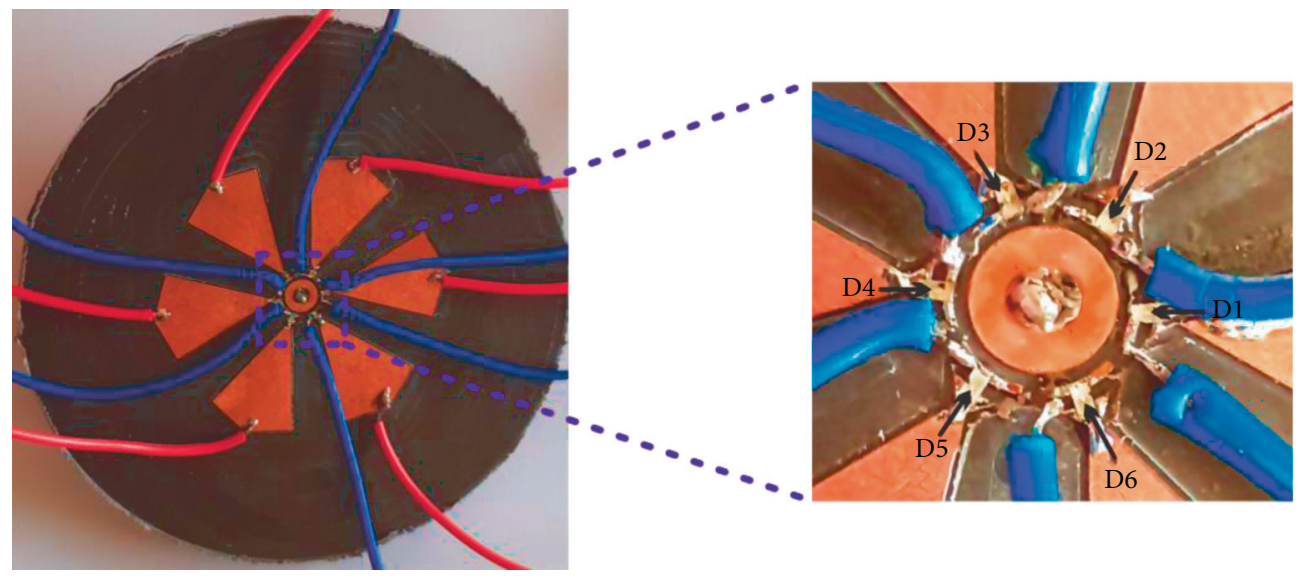

FIGURE 9: Photograph of fabricated antenna.

TABLE 3: Operating states of the proposed antenna.

\begin{tabular}{|c|c|c|c|c|c|c|c|c|c|c|c|}
\hline \multirow{2}{*}{ Operating states } & \multicolumn{6}{|c|}{ PIN diode states } & \multirow{2}{*}{ Center frequency $(\mathrm{GHz})$} & \multirow{2}{*}{ Simulated gain $(\mathrm{dB})$} & \multicolumn{3}{|c|}{ Beam direction } \\
\hline & $D 1$ & $D 2$ & D3 & D4 & D5 & D6 & & & $x y$-plane & $x z$-plane & $y z$-plane \\
\hline State 1 & OFF & OFF & $\mathrm{ON}$ & $\mathrm{ON}$ & $\mathrm{ON}$ & OFF & 5.82 & 9.57 & $\phi=0^{\circ}$ & $\theta=20^{\circ}$ & $\theta=0^{\circ}$ \\
\hline State 2 & OFF & OFF & OFF & $\mathrm{ON}$ & $\mathrm{ON}$ & $\mathrm{ON}$ & 5.82 & 9.57 & $\phi=60^{\circ}$ & $\theta=16^{\circ}$ & $\theta=19^{\circ}$ \\
\hline State 3 & ON & OFF & OFF & OFF & $\mathrm{ON}$ & $\mathrm{ON}$ & 5.82 & 9.57 & $\phi=120^{\circ}$ & $\theta=-15^{\circ}$ & $\theta=19^{\circ}$ \\
\hline State 4 & $\mathrm{ON}$ & $\mathrm{ON}$ & OFF & OFF & OFF & $\mathrm{ON}$ & 5.82 & 9.57 & $\phi=180^{\circ}$ & $\theta=-20^{\circ}$ & $\theta=0^{\circ}$ \\
\hline State 5 & $\mathrm{ON}$ & $\mathrm{ON}$ & $\mathrm{ON}$ & OFF & OFF & OFF & 5.82 & 9.57 & $\phi=240^{\circ}$ & $\theta=-15^{\circ}$ & $\theta=-19^{\circ}$ \\
\hline State 6 & OFF & ON & ON & ON & OFF & OFF & 5.82 & 9.57 & $\phi=300^{\circ}$ & $\theta=16^{\circ}$ & $\theta=-19^{\circ}$ \\
\hline
\end{tabular}

antenna. Thebandwidth of the measured result is $9.07 \%$ (from 5.58 to $6.11 \mathrm{GHz}$ ). While forthe simulated values, the bandwidth is $9.62 \%$ (from 5.54 to $6.10 \mathrm{GHz}$ ). Further,the theoretical value is $9.31 \%$ (from 5.53 to $6.07 \mathrm{GHz}$ ). It is observed that the results are in good agreement. The reasons behind this improved bandwidth are the employment of electronic switches with smaller capacities and the use of fan-shaped coupling elements as shown in Figure 3. The small disagreement between simulated and measured results can be ascribed to tolerances in fabrication process. All the results presented are given when the antenna is operating in state 1 . The same results are obtained for the other operating states.

The antenna efficiency increases from $85 \%$ to $95 \%$ with increasing frequency through the operating range $(5.54-6.10 \mathrm{GHz})$, as shown in Figure 10(b). The designed antenna has a maximum gain of $9.57 \mathrm{~dB}$ and a maximum directivity of $10.07 \mathrm{~dB}$ for all pattern states. The reasons 


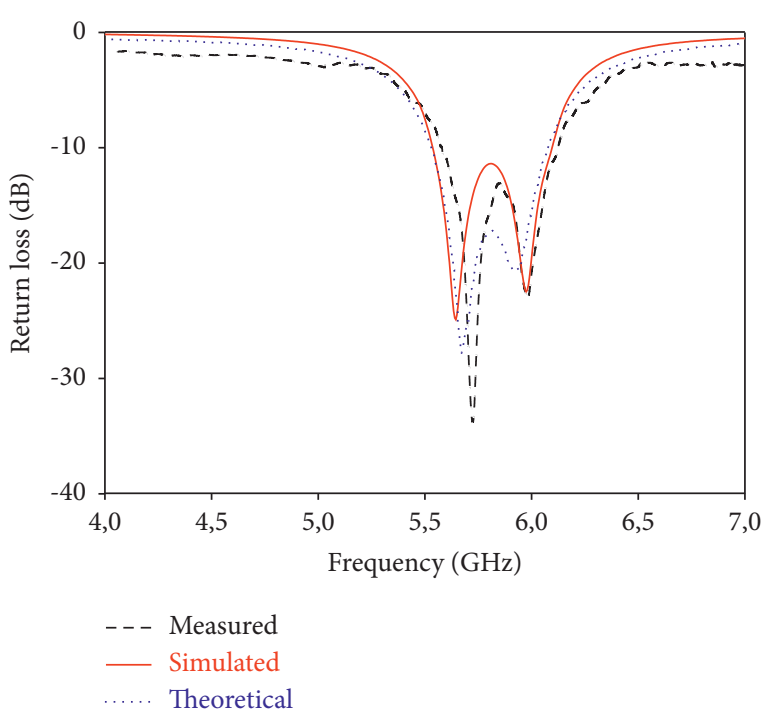

(a)

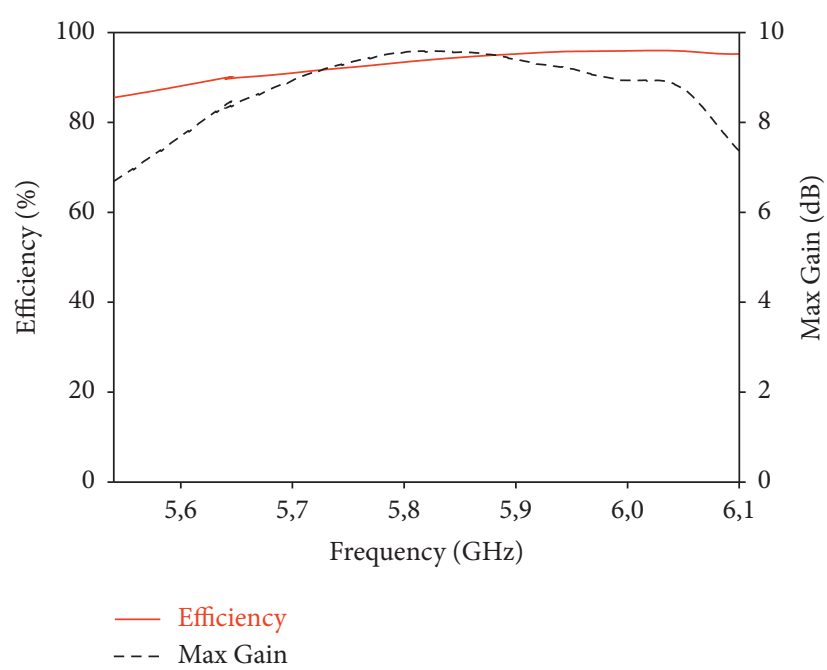

(b)

Figure 10: (a) Simulated, theoretical, and measured S11 and (b) simulated antenna efficiency and gain.

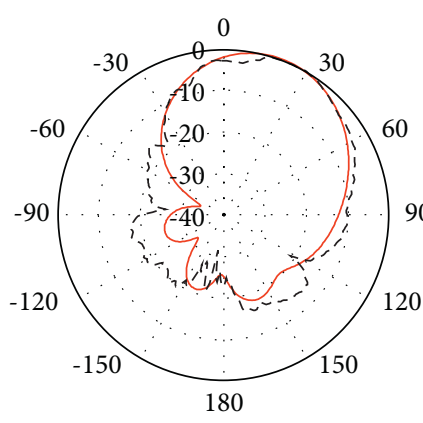

— Simulated

- - - Measured

(a)

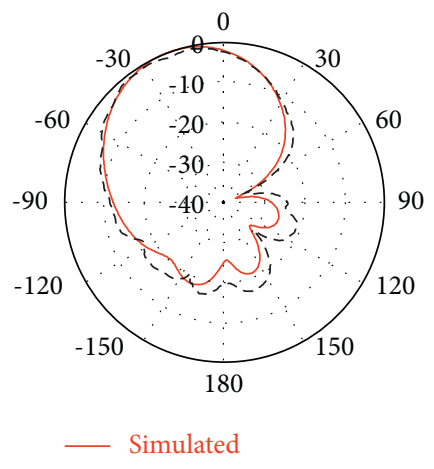

- - - Measured

(d)

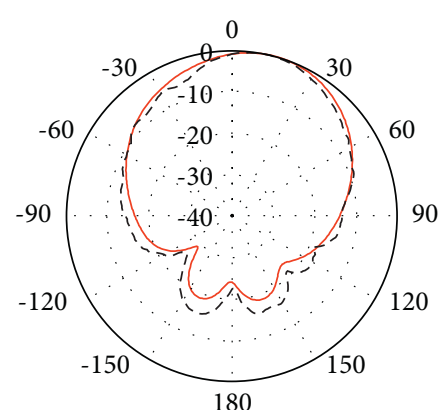

- Simulated

- - - Measured

(b)

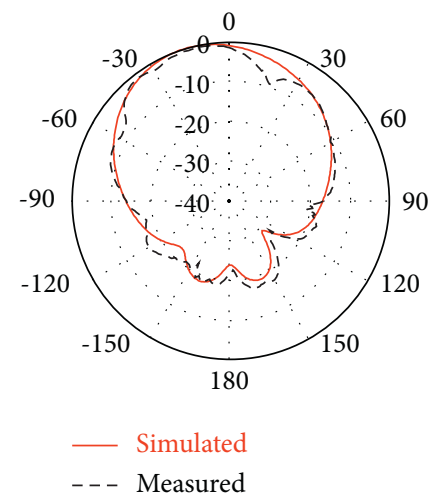

(e)

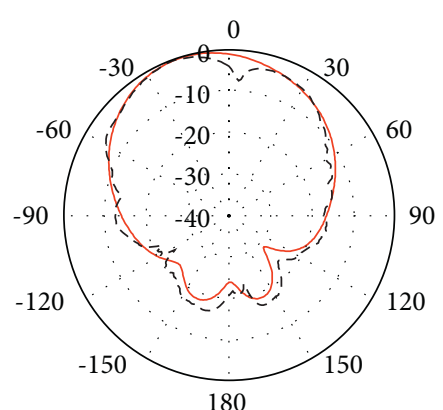

— Simulated

- - - Measured

(c)

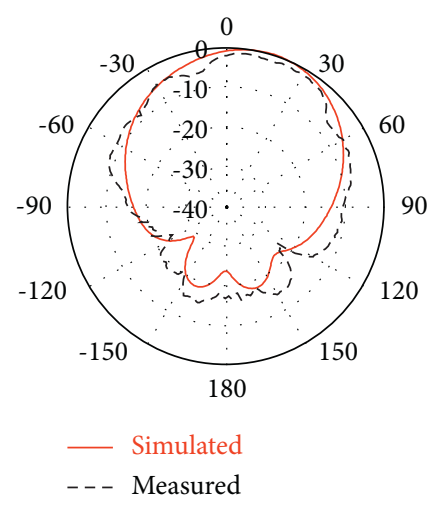

(f)

FIGURE 11: Simulated and measured radiation patterns of the proposed antenna in the $x z$-plane $\left(\phi=0^{\circ}\right)$ at $5.82 \mathrm{GHz}$ : (a) State 1, (b) State 2, (c) State 3, (d) State 4, (e) State 5, and (f) State 6.

behind such high efficiency and enhanced antenna gain in this work are the use, for each state, of three radiating cells as directors and three other radiating cells as reflectors and the use of a low loss dielectric of Rogers RT5880 with loss tangent of 0.0009 .
Far-field radiation patterns of the presented antenna are measured by an antenna measurement system of Geozondas Ltd. Figures 11(a)-11(f) show the simulated and measured $x z$-plane $\left(\phi=0^{\circ}\right)$ radiation patterns. The antenna's main beam can be steered to approximately $20^{\circ}$ (right endfire), $16^{\circ}$ 


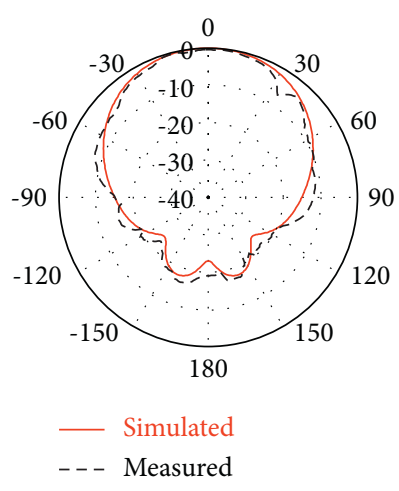

(a)

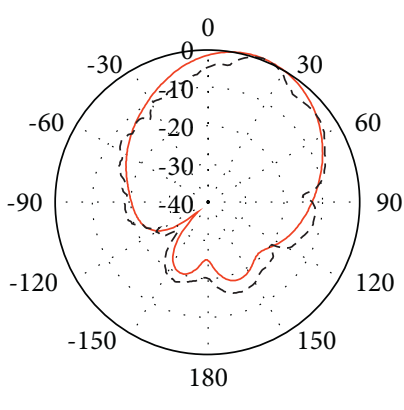

- Simulated

- - - Measured

(c)

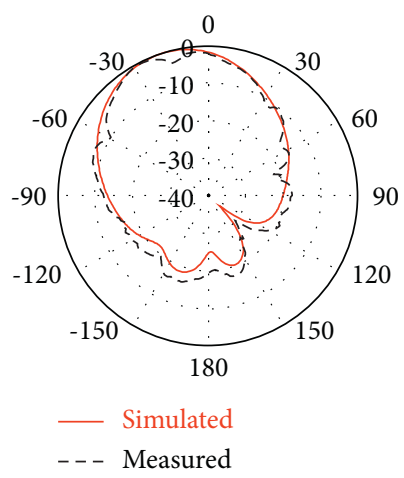

(e)

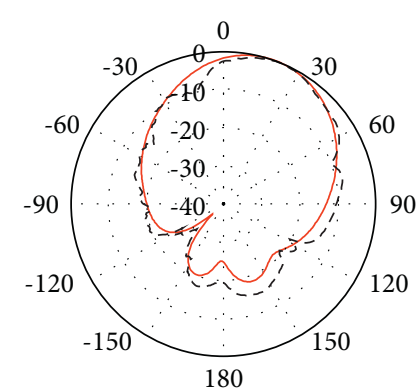

- Simulated

- - Measured

(b)

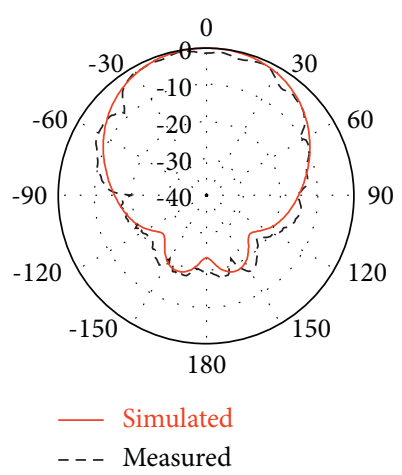

(d)

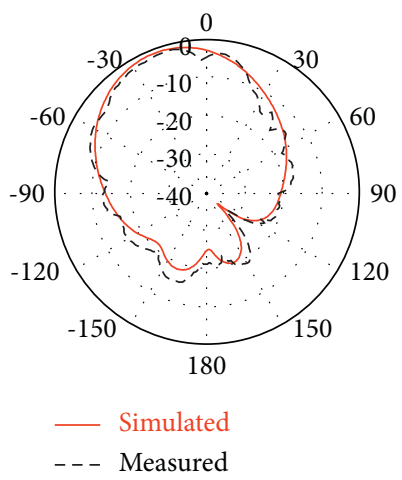

(f)

FIGURE 12: Simulated and measured radiation patterns of the proposed antenna in the $y z$-plane $\left(\phi=90^{\circ}\right)$ at 5.82 GHz: (a) State 1, (b) State 2, (c) State 3, (d) State 4, (e) State 5, and (f) State 6.

(right endfire), $-15^{\circ}$ (left endfire), $-20^{\circ}$ (left endfire), $-15^{\circ}$ (left endfire), and $16^{\circ}$ (right endfire), for State 1 to State 6, respectively.

Figures 12(a)-12(f) present the simulated and measured $y z$-plane $\left(\phi=90^{\circ}\right)$ radiation patterns. The antenna's main beam can be steered to approximately $0^{\circ}$ (boresight), $19^{\circ}$ (right endfire), $19^{\circ}$ (right endfire), $0^{\circ}$ (boresight), $-19^{\circ}$ (left endfire), and $-19^{\circ}$ (left endfire), for State 1 to State 6, respectively. Far-field radiation patterns at frequency of $5.82 \mathrm{GHz}$ in $x z$-plane and $y z$-plane show that proposed reconfigurable pattern antenna radiates in six different directions. The measured results agree well with the simulated predictions, which validate the antenna operation.
Figure 13 shows simulated $3 \mathrm{D}$ radiation patterns at $5.82 \mathrm{GHz}$. The antenna's main beam can be steered in $x y$ plane (azimuth plane) to approximately $0^{\circ}, 60^{\circ}, 120^{\circ}, 180^{\circ}$, $240^{\circ}$, and $300^{\circ}$, for State 1 to State 6 , respectively. Far-field radiation patterns at frequency $5.82 \mathrm{GHz}$ in the azimuth plane show that proposed antenna radiates in six different directions.

To validate the proposed design, Table 4 displays a comparison between our reconfigurable antenna and other previously reported designs. The proposed antenna not only has pattern reconfigurability and enhanced gain but also possesses additional advantages, notably an improved impedance bandwidth, a smaller size, and a very high efficiency. 


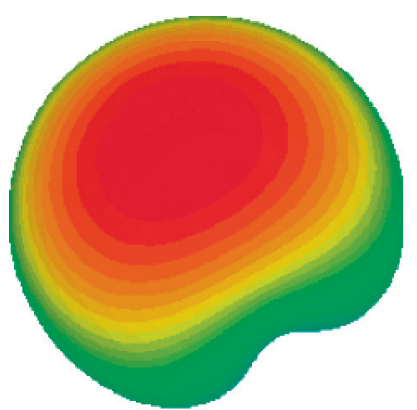

(c)

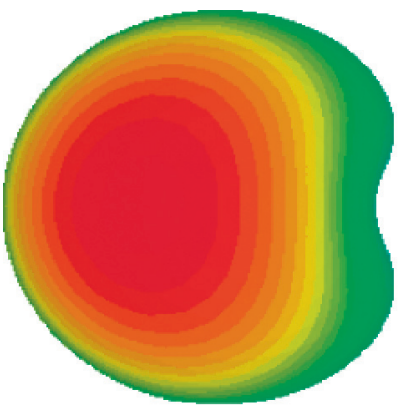

(d)

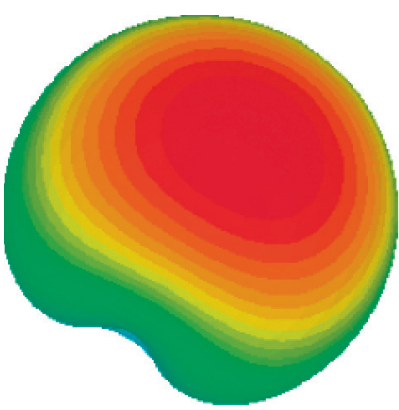

(b)

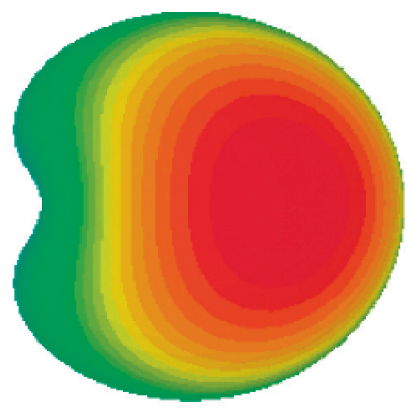

(a)

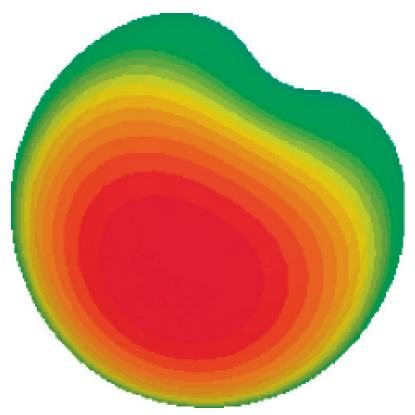

(e)

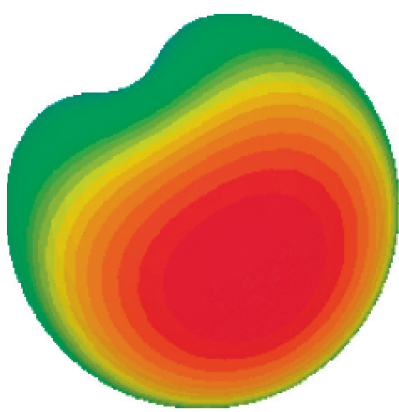

(f)

FIgURE 13: Simulated 3D radiation patterns at $5.82 \mathrm{GHz}$.

TABLE 4: Comparison between the proposed reconfigurable antenna and several other reconfigurable antennas.

\begin{tabular}{lcccccc}
\hline Year/Ref & No. of layers & Size $\left(\lambda_{0}{ }^{3}\right)$ & Frequency $(\mathrm{GHz})$ & Impedance BW $(\%)$ & Gain & Efficiency $(\%)$ \\
\hline $2017 /[19]$ & 2 & $1.17 \times 1.17 \times 0.08$ & 3.4 & 5.6 & 18.6 & $5.4-8.31 \mathrm{dBi}$ \\
$2017 /[28]$ & 1 & $1.37 \times 1.37 \times 0.02$ & 5.9 & $9.3-12.6$ & $6.6 .5 \mathrm{dBi}$ & $6.5 \mathrm{dBi}$ \\
$2018 /[29]$ & 3 & $0.79 \times 0.79 \times 0.28$ & 2.4 & 10.25 & $5.9 \mathrm{dBi}$ & 85 \\
$2019 /[30]$ & 1 & $1,48 \times 1,48 \times 0,008$ & 2.34 & 4.1 & $5.7-6.9 \mathrm{dBi}$ & 89 \\
$2020 /[31]$ & 1 & $0.91 \times 0.85 \times 0.02$ & 3.65 & 12.24 & $7.0-8.9 \mathrm{dBi}$ & NG \\
$2020 /[32]$ & 4 & $1.39 \times 1.39 \times 0.06$ & 2.45 & 9.62 & $9.57 \mathrm{dBi}$ \\
This work & 1 & $0.81 \times 0.81 \times 0.03$ & 5.82 & & 95 \\
\hline
\end{tabular}

$\lambda_{0}$ is the wavelength of the free space.

\section{Conclusion}

In this work, a new antenna with reconfigurable radiation pattern using six fish-shaped radiation cells is simulated, measured, and investigated. Based on the PIN diode switch biasing, the proposed reconfigurable antenna can function at six basic different states and attain radiation pattern reconfigurable characteristic. Changing the distribution of the antenna surface current will deflect the direction of the antenna radiation in six different directions in elevation plane. It indicates that this novel antenna can cover the whole azimuth plane. The designed reconfigurable antenna can provide a wideband ranging from $5.54 \mathrm{GHz}$ to $6.10 \mathrm{GHz}$ centered on $5.82 \mathrm{GHz}$, a maximum gain of $9.57 \mathrm{~dB}$, and a very good efficiency of $95 \%$. It is known that this band is accurately reserved for VANET, Wi-Fi, and WiMAX 
wireless communication systems. Thus, the proposed antenna achieves the goal of this work perfectly.

\section{Data Availability}

The data generated during this study are included within this article.

\section{Conflicts of Interest}

The authors declare that there are no conflicts of interest regarding the publication of this paper.

\section{Acknowledgments}

The authors would like to appreciate Dr Mohammed Ali Ennasar from LaSIT Laboratory, Faculty of Sciences, Abdelmalek Essaadi University, Tetuan, Morocco, for his fruitful guidance and his professional support with regard to the measurement of the proposed antenna.

\section{References}

[1] Z. Mahlaoui, E. Antonino-Daviu, A. Latif, and M. FerrandoBataller, "Design of a dual-band frequency reconfigurable patch antenna based on characteristic modes," International Journal of Antennas and Propagation, vol. 2019, Article ID 4512532, 12 pages, 2019.

[2] S. M. Saeed, C. A. Balanis, and C. R. Birtcher, "Inkjet-printed flexible reconfigurable antenna for conformal WLAN/ WiMAX wireless devices," IEEE Antennas and Wireless Propagation Letters, vol. 15, pp. 1979-1982, 2016.

[3] J. Kim and Y. Sung, "Dual-band microstrip patch antenna with switchable orthogonal linear polarizations," Journal of Electromagnetic Engineering and Science, vol. 18, no. 4, pp. 215-220, 2018.

[4] M. Alibakhshikenari, B. S. Virdee, P. Shukla et al., "Metamaterial-Inspired antenna array for application in Microwave breast imaging systems for tumor detection," IEEE Access, vol. 8, pp. 174667-174678, 2020.

[5] M. A. Kenari and M. N. Moghadasi, "UWB miniature antenna based on the CRLH-TL with increase gain for electromagnetic requirements," Advanced Electromagnetics, vol. 3, no. 1, pp. 61-65, 2014.

[6] I. Kim, B.-C. Park, and J.-H. Lee, "Varactor diode integrated dipole-EBG base-station antenna: enhancing tilted radiation pattern," Microwave and Optical Technology Letters, vol. 57, no. 8, pp. 1794-1799, 2015.

[7] W. D. MA, G. M. Wang, Y. W. Wang, and B. F. Zong, "Compact microstrip antenna with pattern-reconfigurable characteristic," Radioengineering, vol. 26, no. 3, pp. 662-667, 2017.

[8] H. Li, M. Wu, S. Yuan, and C. Zhou, "Design of off-center fed windmill loop for pattern reconfiguration," IEEE Antennas and Wireless Propagation Letters, vol. 18, no. 2, pp. 1626-1630, 2019.

[9] G. Jin, M. Li, D. Liu, and G. Zeng, "A simple planar patternreconfigurable antenna based on arc dipoles," IEEE Antennas and Wireless Propagation Letters, vol. 17, no. 9, pp. 1664-1668, 2018.

[10] S.-L. Chen, P.-Y. Qin, W. Lin, and Y. J. Guo, "Patternreconfigurable antenna with five switchable beams in elevation plane," IEEE Antennas and Wireless Propagation Letters, vol. 17, no. 3, pp. 454-457, 2018.

[11] Y. Tawk, J. Costantine, F. Makhlouf, M. Nassif, L. Geagea, and C. G. Christodoulou, "Wirelessly automated reconfigurable antenna with directional selectivity," IEEE Access, vol. 5, pp. 802-811, 2017.

[12] M.-C. Tang, B. Zhou, Y. Duan, X. Chen, and R. W. Ziolkowski, "Pattern-reconfigurable, flexible, wideband, directive, electrically small near-field resonant parasitic antenna," IEEE Transactions on Antennas and Propagation, vol. 66, no. 5, pp. 2271-2280, 2018.

[13] A. Ayman, "Althuway b-on-chip antenna design using the concepts of metamaterial and SIW principles applicable to terahertz integrated circuits operating over 0.6-0.622 THz," International Journal of Antennas and Propagation, vol. 2020, Article ID 6653095, 9 pages, 2020.

[14] M. Alibakhshikenari, F. Babaeian, B. S. Virdee et al., "A comprehensive survey on "various decoupling mechanisms with focus on metamaterial and metasurface principles applicable to SAR and MIMO antenna systems"," IEEE Access, vol. 8, pp. 192965-193004, 2020.

[15] A. Ayman, "Althuwayb, "Enhanced radiation gain and efficiency of a metamaterial-inspired wideband microstrip antenna using substrate integrated waveguide technology for sub-6 GHz wireless communication systems"," Microwave and Optical Technology Letters, vol. 63, no. 7, pp. 1-7, 2021.

[16] M. Alibakhshikenari, B. S. Virdee, and P. Shukla, "Antenna mutual coupling suppression over wideband using embedded periphery slot for antenna arrays," Electronics, vol. 7, no. 9, 2018.

[17] M. S. Khan, A. Iftikhar, A.-D. Capobianco, R. M. Shubair, and B. Ijaz, "Pattern and frequency reconfiguration of patch antenna using PIN diodes," Microwave and Optical Technology Letters, vol. 59, no. 9, pp. 2180-2185, 2017.

[18] X. He, P. Gao, Z. Zhu, S. You, and P. Wang, "A flexible pattern reconfigurable antenna for WLAN wireless systems," Journal of Electromagnetic Waves and Applications, vol. 33, no. 6, pp. 782-793, 2019.

[19] W.-Q. Deng, X.-S. Yang, C.-S. Shen, J. Zhao, and B.-Z. Wang, "A dual-polarized pattern reconfigurable yagi patch antenna for microbase stations," IEEE Transactions on Antennas and Propagation, vol. 65, no. 10, pp. 5095-5102, 2017.

[20] F. Rahmani, N. A. Touhami, A. B. Kchairi, and N. Taher, "Circular planar antenna with reconfigurable radiation pattern using PIN diodes," Procedia Manufacturing, vol. 46, pp. 760-765, 2020.

[21] F. Rahmani, N. A. Touhami, A. B. Kchairi, N. Taher, and M. El Ouahabi, "Reconfigurable radiation pattern antenna using kite-shaped parasitic patches for wireless access applications," International Journal of Microwave and Optical Technology, vol. 15, no. 2, pp. 187-195, 2020.

[22] F. Rahmani, N. Touhami, A. Kchairi, and N. Taher, "Wideband reconfigurable antenna with beams switching for wireless systems applications," Proceedings, vol. 63, no. 1, p. $36,2020$.

[23] F. Rahmani, N. A. Touhami, A. B. Kchairi, and N. Taher, "Reconfigurable radiation pattern antenna with eight switchable beams in azimuth plane for wlan wireless system," in Proceedings of the 2020 International Conference On Intelligent Systems and Computer Vision (ISCV), Glasgow, Scotland, August 2020.

[24] Datasheet of Microsemi MPP4203, PIN Diodes: http://www. microsemi.com. 
[25] C. A. Balanis, Antenna Theory: Analysis and Design, WileyInterscience, Hoboken, NJ, USA, 2005.

[26] R. Garg, P. Bhartia, I. Bahl, and A. Ittipiboon, Microstrip Antenna Design Handbook, Artech House, Boston, London, 2003.

[27] A. B. Kchairi, M. Boussouis, and N. A. Touhami, "Highperformance LPF using coupled C-shape DGS and radial stub resonators for microwave mixer," Progress In Electromagnetics Research Letters, vol. 58, pp. 97-103, 2016.

[28] Y.-F. Cheng, X. Ding, B.-Z. Wang, and W. Shao, “An azimuthpattern-reconfigurable antenna with enhanced gain and front-to-back ratio," IEEE Antennas and Wireless Propagation Letters, vol. 16, pp. 2303-2306, 2017.

[29] J.-S. Row and Y.-H. Wu, "Pattern reconfigurable slotted-patch array," IEEE Transactions on Antennas and Propagation, vol. 66, no. 3, pp. 1580-1583, 2018.

[30] S. J. Lee, W. S. Yoon, and S. M. Han, "Planar beam steerable parasitic array antenna system design based on the yagi-uda design method," International Journal of Antennas and Propagation, vol. 2019, Article ID 8023712, 9 pages, 2019.

[31] S. Gaya, R. Hussain, M. S. Sharawi, and H. Attia, "Pattern reconfigurable Yagi-Uda antenna with seven switchable beams for WiMAX application," Microwave and Optical Technology Letters, vol. 62, no. 3, pp. 1329-1334, 2020.

[32] L. Kang, H. Li, J. Zhou, and S. Zheng, "An OAM-mode reconfigurable array antenna with polarization agility," IEEE Access, vol. 8, pp. 40445-40452, 2020. 